\title{
EFECTOS DE DOS SISTEMAS DE LABRANZA SOBRE POBLACIONES DE ARROZ ROJO (Oryza sativa L.) EN UN AGROECOSISTEMA ARROCERO INUNDADO ${ }^{1}$
}

\author{
Yemel M. Ortega ${ }^{2}$, Renán Agüero Alvarado 3
}

\begin{abstract}
RESUMEN
Efectos de dos sistemas de labranza sobre poblaciones de arroz rojo (Oryza sativa $L$.) en un agroecosistema arrocero inundado. Se evaluó la influencia de dos sistemas de labranza sobre poblaciones de arroz rojo (Oryza sativa L.) y de otras malezas frecuentes en agroecosistemas arroceros durante cuatro ciclos consecutivos de cultivo comercial de arroz inundado. Para estimar el tamaño del banco de semillas se tomaron muestras de suelo a tres profundidades $(0$ a 5, 5-20 y $20-40 \mathrm{~cm}$ ) al inicio de cada ciclo de cultivo. También se estimó la densidad de las malezas más abundantes emergidas dentro del área en cultivo. En ambos casos, hubo diferencias significativas entre los sistemas de siembra evaluados. Mientras que el banco de semillas de arroz rojo y sus poblaciones emergidas en el campo presentaron tendencias crecientes en labranza mecanizada convencional (LMC), se observó una reducción progresiva en el tamaño del banco y menor densidad, a medida que transcurrieron ciclos sin labranza en siembra directa sobre rastrojos (SDR). Las poblaciones de ciperáceas anuales como Cyperus spp. y Fimbristylis milliaceae incrementaron en ambos sistemas de siembra, pero con mayor intensidad en LMC. La distribución vertical del banco de semillas de malezas en el suelo también fue diferente entre sistemas de siembra. En SDR más del $66 \%$ del banco de semillas de arroz rojo y el $82 \%$ de las semillas que germinaron se encontraron en los primeros cinco cm del perfil del suelo. Los resultados pemitieron concluir que el sistema SDR promueve condiciones propicias para mantener control en las poblaciones de arroz rojo y de otras malezas en el cultivo de arroz inundado.
\end{abstract}

Palabras claves: arroz inundado, malezas, arroz rojo (Oryza sativa L.), rastrojos, labranza convencional, siembra directa.

\begin{abstract}
Effects of two tillage systems on red rice (Oryza sativa L.) populations in a flooded rice agroecosystem. The influence of two tillage systems was evaluated on red rice (Oryza sativa L.) populations and other frequent weeds in rice agroecosystems throughout four consecutive cycles of commercial flooded rice. In order to estimate the seed bank size, soil samples from three depths ( 0 to 5, 5 to 20 and 20 to $40 \mathrm{~cm}$ ) were taken at the beginning of each crop cycle. The density of the most abundant weeds in the crop area was also measured. In both tillage systems there were significant differences in emerged weeds and seed banks. While the red rice seed bank and its emerged populations on the field presented incremental trends in conventional mechanized sowing (CMS), a progressive decrease in bank size and a lower density was observed in direct seeding into stubble (DSS), during cycles in which no tillage was used. Populations of annual sedges like Cyperus spp. and Fimbristylis milliaceae increased in both tillage systems, with greater intensity in CMS. The vertical distribution of the weed seed bank in the soil was also different for both tillage systems. In DSS more than $66 \%$ of the red rice seed bank and $82 \%$ of the germinated seeds were found within the first five $\mathrm{cm}$ of the soil profile. It is concluded that the DDS system promotes favorable conditions to maintain control over red rice populations and other weeds in flooded rice.
\end{abstract}

Key words: Flooded rice, weeds, red rice (Oryza sativa L.), stubbles, conventional tillage, direct sowing.

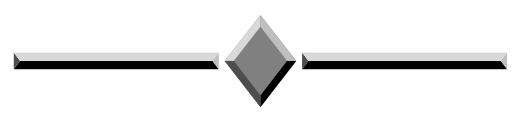

\footnotetext{
1 Recibido: 11 de noviembre, 2004. Aceptado: 18 de marzo, 2005. Parte de la Tesis de doctorado del primer autor, en Sistemas de Producción Agrícola Tropical Sostenible, Universidad de Costa Rica.

2 Programa de Doctorado en Sistemas de Producción Agrícola Tropical Sostenible, Universidad de Costa Rica; yemel.ortega@edifystudios.com

3 Facultad de Ciencias Agroalimentarias, Universidad de Costa Rica; raguero@cariari.ucr.ac.cr
} 


\section{INTRODUCCIÓN}

Se denomina "arroz rojo" a algunas poblaciones de especies del género Oryza que se comportan como malezas en los agroecosistemas cultivados con arroz comercial. Esta maleza se localiza en todas las grandes regiones arroceras tropicales y es de manera particular un serio problema en la siembra directa de arroz en Latino y Norte América, el Caribe y África, así como en el sur y sureste de Asia (Mortimer et al. 2000). En Costa Rica, el arroz rojo incluye las especies $O$. sativa $f$. spontanea (propiamente arroz rojo) y O. latifolia (arroz pato), así como el arroz comercial e híbridos de éste cuando crecen de manera espontánea (Agüero 1996). Dentro del grupo sativa se incluyen los arroces cultivados y ciertas especies del género estrechamente relacionadas (todas con genoma designado como AA); mientras que las del grupo latifolia incluye sólo especies que son consideradas malezas y con genomas ${ }^{4}$ designados como BB, CC, DD, EE, FF; y BBCC o CCDD para el caso de algunas especies tetraploides (Khush et al. 1999).

$\mathrm{El}$ arroz rojo es generalmente un competidor superior a los cultivares comerciales, debido a su vigor temprano, mayor ahijamiento, mayor tamaño de plantas y capacidad de autodesgrane. Las semillas pueden presentar dormancia y mantener su viabilidad por muchos años, lo cual facilita su persistencia como maleza en los subsecuentes ciclos de cultivo. En las primeras etapas de crecimiento las plántulas de arroz rojo son muy similares a las del cultivo. Después de la formación de panículas el reconocimiento se facilita por la distinta pigmentación de las aristas y el pericarpio (desde negro y rojo oscuro, hasta blanco), así como la mayor dehiscencia de sus granos.

La alta elasticidad del proceso de germinación de semillas de arroz rojo, caracterizada por su habilidad para germinar antes del cultivo y escaparse a los tratamientos para su control, favorece su capacidad competitiva. Históricamente la forma perjudicial más conocida de arroz rojo es Oryza sativa L., caracterizada por tener cariópsides con pericarpios pigmentados. El nivel de daño que ocasiona sobre el cultivo es variable, y depende de la capacidad competitiva de cada biotipo de maleza, variedad o híbrido presentes en el agroecosistema. En terrenos infestados por arroz rojo, se han detectado daños por competencia a partir de dos plantas por metro cuadrado (Kwon et al. 1991). El daño de una

\footnotetext{
4 Las especies de Oryza que tienen el genoma "A" pueden cruzarse con mayor facilidad con el arroz cultivado, y por esto han sido usadas como donantes de genes para conferir protección contra insectos y enfermedades. Las especies del grupo latifolia por poseer genomas distintos no pueden aparearse naturalmente con las del grupo sativa.
}

sola planta de arroz rojo equivale al efecto de cuatro plantas del cultivo en competencia intraespecífica (Pantone y Baker 1991).

Smith (1988) reportó que una competencia temprana de infestaciones relativamente bajas (10 - 20 plantas $/ \mathrm{m}^{2}$ ) de arroz rojo puede ocasionar pérdidas en producción del 50\%. En Latinoamérica se ha estimado que 24 plantas $/ \mathrm{m}^{2}$ pueden causar pérdidas de rendimiento del 75\% (Fisher y Ramírez 1993). El arroz rojo al acamarse causa pérdidas por volcamiento del cultivo, constituye una maleza en cultivos alternos, su control es costoso, y se requieren varios períodos de siembra o largas rotaciones para su eliminación (González 1985). Los costos directos (mano de obra y herbicidas) para el control de arroz rojo y otras malezas en arroz varían entre 5\% y $13 \%$ de los costos totales de producción (Moody 1991). En adición a estos costos directos, los costos indirectos del control de malezas incluyen los de preparación de tierra y manejo del agua llevados a cabo específicamente para controlar malezas, daños fitotóxicos al cultivo y posibles efectos adversos a la salud humana o al ambiente. Además de las pérdidas en el campo, la amplia presencia de granos rojos comercialmente indeseables requiere una intensiva industrialización para remover el pericarpio, lo que resulta en una mayor proporción de granos partidos y menor rendimiento final de grano entero (Baker y Sonnier 1983).

Debido a los daños que ocasiona el arroz rojo es necesario considerar programas de manejo que conduzcan a su erradicación de los campos cultivados con arroz. Para erradicar cualquier maleza es preciso que la población continuamente decline a lo largo de ciclos sucesivos, hasta llegar a agotar las reservas del banco de semillas del suelo. Esta tarea resulta más difícil si se considerara al arroz comercial espontáneo como maleza.

El control de arroz rojo es difícil por su estrecha relación taxonómica con el arroz comercial y lo complicado de aplicar medidas que seleccionen diferencias entre poblaciones física y fisiológicamente muy parecidas. Al diseñar un programa de control de arroz rojo es relevante tener un enfoque ecológico integral para prevenir la infestación de los campos. Debido a la longevidad de las semillas de arroz rojo ${ }^{5}$ debe evitarse la

\footnotetext{
5 La longevidad de la semilla en el suelo depende del ecotipo y es afectada por la profundidad a la cual está enterrada, el tipo de suelo, la humedad y la intensidad de la dormancia. Experimentos desarrollados por Nolding (1995) y Zorrilla y Acevedo (1996) indican que semillas de arroz rojo a profundidades de $15-25 \mathrm{~cm}$ mantienen mayor viabilidad que las enterradas de $0-5 \mathrm{~cm}$. Tomando en cuenta estos resultados debería reducirse la labranza cuando existen infestaciones de arroz rojo, rotar con otros cultivos o bien realizar agotamientos.
} 
exposición de semillas dormantes a condiciones favorables para su proliferación y la adición al banco de semilla del suelo de semilla superficial procedente del ciclo anterior. Para tales propósitos es importante usar semilla pura ${ }^{6}$ en los nuevos cultivos, y utilizar maquinaria e implementos limpios en las labores de la finca.

La similitud entre el arroz rojo y las variedades comerciales ha impedido la aplicación de herbicidas para controlar esta maleza en forma selectiva. El uso de glifosato, glufosinato de amonio e imazetapir podría ser posible en cultivares modificados mediante biotecnología vegetal para conferir tolerancia a tales herbicidas. Con cultivares de este tipo se podría lograr el control selectivo de arroz rojo y otras malezas en arroz, pero preocupa el posible impacto negativo de su uso comercial por la posible transferencia de genes de resistencia del cultivo al arroz rojo (Olofsdotter et al. 1999). Esta opción sería una buena alternativa si se evita la diseminación de la resistencia a las poblaciones de arroz rojo y las malezas en general, lo cual podría lograrse mediante barreras genéticas para controlar el flujo de genes y reducir la adaptabilidad de las progenies híbridas (Fisher 1999).

\section{Rotación de cultivos}

La rotación de cultivos en combinación con labranza reducida pueden ser formas efectivas de limitar la producción de semillas de malezas gramíneas como el arroz rojo y hojas anchas, independientemente del nivel de insumos aplicado para el manejo (Kegode et al. 1999). Cuando es viable rotar con otros cultivos, especialmente con plantas leguminosas o establecer pasturas que son controladas con aplicaciones totales de herbicidas antes del establecimiento del ciclo siguiente se pueden obtener resultados aceptables. Las rotaciones permiten combinar diferentes atributos de los cultivos (patrones de competencia por los recursos, interferencias alelopáticas, disturbios del suelo y daños mecánicos) para crear un ambiente desfavorable que limita la proliferación de muchas malezas. No obstante esta práctica es útil sólo en condiciones ambientales y económicas particulares.

6 El Reglamento de certificación de semillas de arroz para Costa Rica permite como máximo una semilla de arroz rojo por cada kilogramo de semilla certificada, y dos en caso de semilla autorizada. Una sola semilla de arroz rojo por kilogramo bastaría para producir $50 \mathrm{~kg}$ de semillas de dicha maleza al cabo de tres ciclos consecutivos de cultivo, por lo cual aún con semilla certificada hay probabilidades de infestar áreas libres de esta maleza. Debido a lo prolífero del arroz rojo y a la dificultad de su control, lo mejor sería tener cero tolerancia de semillas de arroz rojo en la semilla comercial del arroz.
En el caso específico del presente estudio la rotación no se consideró una opción viable porque son muy pocos los cultivos alternativos con capacidad para desarrollarse en los suelos pesados e hidromórficos donde crece el arroz en Costa Rica, que tengan además precios competitivos. La infraestructura particular para sistemas de arroz inundado (terrenos nivelados, diques, canales de irrigación y drenaje) debería ser modificada con cada rotación, lo cual incrementaría de manera significativa los costos de producción ${ }^{7}$.

\section{Sistemas de labranza e inundación}

Dentro de las prácticas culturales la labranza puede ser utilizada para promover la emergencia de plántulas de arroz rojo y su posterior eliminación. De este modo se podría agotar gradualmente el banco de semillas. La labranza afecta la distribución vertical de las semillas en el perfil del suelo. En suelos no disturbados, donde no se ha practicado la labranza la mayoría de las semillas de malezas permanece sobre o cerca de la superficie del suelo. La labranza con arado de vertedera entierra las semillas a mayor profundidad dentro del banco de semillas. En un estudio realizado en Ontario por Clements et al. (1996) se encontró que los $5 \mathrm{~cm}$ superficiales del suelo contenían con cero labranza $(74 \%)$, arado de cincel $(61 \%)$, arado de vertedera (37\%). El algunos casos, el demorar la primera labranza después de la cosecha puede influir en la cantidad de semillas que entran al banco. Cuando las semillas de malezas quedan expuestas por más tiempo sobre el suelo su cantidad se ve afectada por altas temperaturas y depredación por pájaros y ratones (Westerman et al. 2003).

La labranza debe ser superficial para no incorporar las semillas en el suelo y favorecer su dormancia. Este es un atributo común de muchas semillas de malezas que dificulta predecir la magnitud y el momento cuando ocurrirá la emergencia de las malezas. Una vez sea removido este impedimento, la germinación de la semilla procedería bajo un amplio rango de condiciones ambientales. Mantener las semillas cerca de la superficie reduce la dormancia, promueve la germinación y permite aplicar estrategias de manejo de malezas más eficientes. En suelos no laborados un adecuado control de malezas emergidas, durante varios años, puede disminuir el banco de semillas en la capa superficial del suelo. Enterrar la semilla profundamente en el suelo complica los esfuerzos futuros de control de malezas (Swanton et al. 2001).

\footnotetext{
7 Fernández, Leonel. 2004. Comunicación personal. Hacienda el Pelón de la Bajura, Costa Rica.
} 
Los sistemas de labranza pueden afectar la dinámica de la desactivación de la dormancia en las poblaciones de semillas de arroz rojo, alterando el tiempo de emergencia y el número de plántulas establecidas. Según Benech-Arnold et al. (2000) dentro de los factores que controlan la desactivación de la dormancia pueden distinguirse los que modifican su nivel o intensidad en la población de semillas (temperatura y condición hídrica del suelo), de los que terminan o eliminan las últimas restricciones para la germinación de la semilla, una vez que el grado de dormancia es suficientemente bajo (luz, fluctuaciones de temperatura, concentración de nitratos).

En el sistema de arroz inundado la anoxia por inundación evita la germinación de arroz rojo (Diarra et al. 1985). El control con agua se basa en que el arroz rojo no puede germinar cuando las semillas están cubiertas con suelo y agua. La germinación puede ocurrir si la semilla sólo esta cubierta por cualquiera de los dos elementos (González 1985). Una lámina de agua sobre el suelo después de sembrar semilla pregerminada del cultivo puede mantener la anaerobiosis sobre el perfil superficial del suelo, impidiendo la germinación de semilla de arroz rojo.

En sistemas agrícolas inundados el ambiente donde las semillas deben germinar está caracterizado por bajas concentraciones de oxígeno. Esta condición desactiva la dormancia en semillas de malezas comunes en el cultivo de arroz como Echinocloa turnerana (Conover y Geiger 1984), y Leersia oryzoides (Rosa y Corbineau 1986). La inundación también ocasiona la muerte de semillas de especies no adaptadas y favorece el desarrollo de las que si lo son. Por ejemplo, en el caso de Ambrosia tenuifolia la inmersión de semillas de esta especie en agua a bajas temperaturas por varias semanas incrementa su sensibilidad a la luz, activándose los procesos de germinación (Insausti et al. 1995). En tal sentido, el fangueo del suelo y trasplante del cultivo en campos inundados ha sido una práctica exitosa, permitiendo la recuperación de campos severamente infestados con arroz rojo, en terrenos donde previamente no era económicamente viable sembrar arroz. Sin embargo, está demostrado que el fangueo además de ser muy costoso resulta perjudicial para el suelo, afectando principalmente algunas características físicas. Además, en América Latina los beneficios que pueden obtenerse de las prácticas para el control de la germinación del arroz rojo por anaerobiosis se ven limitadas por el alto costo de una buena nivelación del terreno y la poca disponibilidad de agua en muchas regiones.
Otro método efectivo consiste en la selección manual de plántulas en el vivero, trasplante y deshierba manual intensiva. Sin embargo, esta práctica resulta viable únicamente cuando existe abundante mano de obra a bajo costo, lo cual cada vez resulta menos común debido al desplazamiento de la fuerza laboral humana a otras actividades económicas distintas a la agricultura (Zhang 2000).

Para cada sistema de labranza que se aplique al cultivo de arroz es preciso identificar los cambios que ocurren en las poblaciones de malezas a fin de ajustar la estrategia para su control. Debido a que en teoría la SDR podría reducir las poblaciones de arroz rojo, es importante estudiar su eficacia en condiciones de campo y los problemas en el manejo de malezas que podrían surgir con su adopción. Si no se toman medidas correctivas a tiempo, el control de malezas podría ser más costoso y complicado, además de ocasionar mayores pérdidas en los rendimientos del cultivo (Buhler et al. 1998). El objetivo de este estudio fue determinar el efecto de los sistemas labranza mecanizada convencional (LMC) y siembra directa sobre rastrojos (SDR) sobre el banco de semillas del suelo y la emergencia de poblaciones de arroz rojo y otras malezas comunes en un agroecosistema de arroz inundado en el Pacífico Norte de Costa Rica.

\section{MATERIALES Y MÉTODOS}

\section{Elección del sitio}

La fase experimental se desarrolló desde diciembre del 2001 a mayo del 2004, en lotes comerciales de arroz inundado de la hacienda El Pelón de la Bajura, en la Región Pacífico Norte de Costa Rica. El experimento principal se instaló en el lote Playitas realizándose evaluaciones en cuatro ciclos consecutivos iniciados el 14 de marzo del 2002, 1 de diciembre del 2002, 23 de julio del 2003 y 26 de diciembre del 2003. Réplicas del mismo fueron instaladas en otros tres lotes adyacentes (Bribri, Cabuyo 2, y José Joaquín). El historial de uso y manejo de estos lotes fue similar durante los 10 últimos años. Antes de iniciar el ensayo el terreno fue nivelado para asegurar una cobertura uniforme con lámina de agua. En cada lote fueron instaladas dos parcelas cuadradas de $4900 \mathrm{~m}^{2}$ de superficie, con un borde de separación de 10 metros de ancho entre las mismas.

El arreglo de los tratamientos se realizó asignando los sistemas: siembra directa sobre rastrojos (SDR) y labranza mecanizada convencional (LMC) a parcelas 
contiguas, las cuales fueron divididas en 49 subparcelas (repeticiones) cuadradas con $100 \mathrm{~m}^{2}$ de área efectiva cada una. Los suelos fueron de textura francoarcillosa (33\% arena, 29\% limo, 38\% arcilla, 3\% de materia orgánica y $\mathrm{pH} 6,1)^{8}$, de color gris oscuro. Durante los ciclos evaluados se cultivó arroz variedad CR1113, establecido con una sembradora marca SEMEATO TDNG 420, calibrada para sembrar $180 \mathrm{~kg} / \mathrm{ha}$ de semilla, con un ancho de siembra de 4,20 m, y accionada por un tractor agrícola de $100 \mathrm{HP}$, a una velocidad de operación aproximada a $7 \mathrm{~km} / \mathrm{h}$. Este equipo de siembra está diseñado para cortar los rastrojos, abrir un pequeño surco en el suelo, colocar la semilla y finalmente cerrar el surco abierto, todo en una sola operación.

\section{Prácticas culturales}

En las parcelas tratadas bajo SDR se efectuó un control de malezas cinco días antes de la siembra. Se aplicó el herbicida glifosato, a razón de $3 \mathrm{~kg} / \mathrm{ha}$ de producto formulado $68 \mathrm{SG}$ en 200 1/ha. El arroz fue sembrado bajo los rastrojos de la cosecha anterior y las malezas desecadas mediante la aplicación del tratamiento herbicida. La aplicación se efectuó a una velocidad de $6 \mathrm{~km} / \mathrm{h}$, utilizando una aspersora mecanizada accionada por un tractor agrícola de $100 \mathrm{HP}$ de potencia. El equipo para la aplicación consistió en un aguilón telescópico con 48 boquillas tipo Turbo T-Jet 8002, distanciadas entre sí a $25 \mathrm{~cm}$, para cubrir $12 \mathrm{~m}$ de ancho por pasada. Este implemento se ajustó a 0,5 m de altura sobre el nivel de las malezas.

En las parcelas bajo LMC el suelo fue preparado mediante una pasada de rastra rompedora, una de rastra afinadora y finalmente un pase de rodillo compactador. Se empleó una rastra con 40 discos de 98 cm de diámetro, tirada por un tractor agrícola con $200 \mathrm{HP}$ de potencia. En la superficie del terreno no quedaron restos visibles de rastrojos ni malezas. Para evitar la emergencia de nuevas malezas se efectuó una aplicación de herbicida preemergente butaclor (4 1/ha de producto formulado $60 \mathrm{EC}$ en 200 1/ha de agua).

El control de plagas así como la fertilización fue igual para ambos sistemas en estudio. Se aplicó nitrógeno a dosis que variaron entre 170 a $200 \mathrm{~kg} / \mathrm{ha}$, suplido en forma de urea y de las fórmulas 21,1-8,0-8,5-3,4 (MgO) y 26-0-26. Las dosis de fósforo, potasio y magnesio fueron de 20,125 y $8 \mathrm{~kg} /$ ha de $\mathrm{P}_{2} \mathrm{O}_{5}, \mathrm{~K}_{2} \mathrm{O}$ y $\mathrm{MgO}$, respectivamente. Se realizaron dos aplicaciones terrestres y tres aéreas.

\footnotetext{
8 Según análisis físicos y químicos realizados en el Laboratorio de Suelos, Centro de Investigaciones Agronómicas (CIA), Universidad de Costa Rica (UCR).
}

\section{Evaluación de malezas emergidas en el campo}

En cada ciclo de cultivo evaluado se realizó un levantamiento de las malezas emergidas en el terreno a 60 días después de la siembra (dds). Para esto se usó un marco metálico de $1 \mathrm{~m}^{2}$, evaluando dos puntos de muestreo escogidos al azar por subparcela. En cada punto de muestreo se registró el tipo de maleza y cantidad de plántulas presentes.

\section{Estimación del banco de semillas del suelo}

Se estimó el tamaño del banco de semillas del suelo y su distribución vertical tanto de arroz rojo como de las malezas más comunes del agroecosistema inundado. Se tomaron muestras de suelo horizontalmente distribuidas al azar, seleccionando un punto de muestreo por subparcela, y haciendo calicatas para tomar muestras de manera sistemática en tres rangos de profundidad; 0 a 5,5 - 20 y 20 - $40 \mathrm{~cm}$, respectivamente. Se colectó un volumen de $1000 \mathrm{cc}$ de suelo por muestra y se depositó en bolsas plásticas, debidamente identificadas.

Las muestras fueron sometidas a dos procedimientos. En el primer procedimiento, las semillas contenidas en $500 \mathrm{cc}$ de suelo por muestra se pusieron a germinar en potes bajo condiciones favorables de humedad, luz y temperatura, dentro de un invernadero. La germinación de semillas de malezas fue evaluada durante seis meses. Periódicamente se extrajeron, identificaron y contaron las plántulas nacidas. Este procedimiento permitió apreciar directamente la densidad de semillas viables que hay en el terreno. Debido a que las semillas fuertemente aletargadas no germinaron, fue imposible considerarlas en esta parte de la investigación.

El segundo procedimiento consistió en separar las semillas mecánicamente, lo cual se hizo lavando las muestras sobre tamices con mallas de 1,5, 1,0 y 0,5 mm de luz, adecuados para retener semillas de distinto tamaño sin perder las más pequeñas. Las semillas retenidas fueron secadas a temperatura ambiente, para luego separarlas manualmente e identificarlas bajo un estereoscopio. Se evaluó el estado físico de las semillas para diferenciar semillas viables de semillas deterioradas y muertas.

\section{Análisis estadístico}

Se realizaron pruebas de igualdad de medias mediante el estadístico t-student para contrastar las variables en estudio del banco de semillas por sistema de siembra y profundidad de muestreo, germinación de semillas en potes por profundidad de muestreo, así como 
tipo y frecuencia de malezas emergidas en el campo por sistema de siembra.

\section{RESULTADOS Y DISCUSIÓN}

Los resultados se dividen en seis secciones correspondientes a los principales experimentos de la investigación. Primero se describe la composición de las malezas, contrastando su abundancia relativa en ambos sistemas de labranza. Luego se analizan los cambios en la densidad de gramíneas, ciperáceas y hojas anchas anuales en cada sistema y se presenta una estimación del tamaño del banco de semillas del suelo. En la sección de malezas emergidas en el campo, se relaciona la cantidad de malezas emergidas con el banco de semillas del suelo. Finalmente, se contrasta la distribución vertical del banco de semillas de arroz rojo entre los dos sistemas de labranza en estudio.

\section{Principales malezas presentes}

Todas las malezas identificadas durante los cuatro ciclos de cultivo evaluados fueron anuales. El Cuadro 1 presenta la composición relativa del banco de semillas del suelo (0 - $40 \mathrm{~cm}$ de profundidad), la fracción de plántulas de malezas germinadas en potes en condiciones de invernadero y la abundancia relativa de malezas detectadas en el campo; expresadas como porcentajes del total de semillas o plantas identificadas. Estos datos reflejan la composición del banco de semillas y malezas emergidas en campo luego de tres ciclos consecutivos de cultivo. Con estos datos, se compara en forma relativa el efecto de los dos sistemas de preparación del terreno al momento de la siembra, sobre la densidad de malezas que se establecen en competencia con el cultivo de arroz. Las especies de malezas más importantes fueron agrupadas en gramíneas (arroz rojo, arrocillo, guarda rocío, pata de gallina y plumilla); ciperáceas (con representación de los géneros Cyperus y

Cuadro 1. Tamaño relativo del banco de semillas del suelo*, cantidad de semillas germinadas en invernadero y plántulas emergidas en el campo, según tipo de maleza y sistema de siembra. Hacienda El Pelón de la Bajura, Guanacaste, Costa Rica. 2004.

\begin{tabular}{|c|c|c|c|c|c|c|c|c|}
\hline \multirow{3}{*}{$\begin{array}{c}\text { Grupo } \\
\text { Familia } \\
\text { botánica }\end{array}$} & \multirow{3}{*}{ Nombre científico } & \multirow{3}{*}{ Nombre común } & \multicolumn{6}{|c|}{ Semillas y plántulas germinadas o emergidas } \\
\hline & & & \multicolumn{2}{|c|}{ Banco } & \multicolumn{2}{|c|}{ Invernadero } & \multicolumn{2}{|c|}{ Campo } \\
\hline & & & SDR & LMC & SDR & LMC & SDR & LMC \\
\hline \multicolumn{9}{|l|}{ Gramíneas } \\
\hline \multirow[t]{5}{*}{ Poaceae } & Digitaria sanguinalis (L.) Scop. & Guarda rocío & 2 & 1 & 1 & 1 & 2 & 2 \\
\hline & Echinochloa colona (L.) Link. & Arrocillo & 9 & 6 & 3 & 1 & 9 & 10 \\
\hline & $\begin{array}{l}\text { Eleusine indica (L.) Gaertner } \\
\text { Leptochloa filiformis (Pers.) P. }\end{array}$ & Pata de gallina & 3 & 1 & 3 & 1 & 4 & 2 \\
\hline & Beauv. & Plumilla & 3 & 1 & 3 & 2 & 4 & 2 \\
\hline & Oryza sativa $\mathrm{L}$. & Arroz rojo & 28 & 36 & 19 & 27 & 19 & 31 \\
\hline \multicolumn{9}{|l|}{ Ciperáceas } \\
\hline \multirow[t]{2}{*}{ Cyperaceae } & Cyperus spp. & Coyolillos & 26 & 27 & 40 & 41 & 18 & 21 \\
\hline & Fimbristylis milliaceae & Pelo de chino & 12 & 13 & 18 & 21 & 12 & 10 \\
\hline \multicolumn{9}{|l|}{ Hojas anchas } \\
\hline \multirow[t]{2}{*}{ Asteraceae } & Eclipta alba (L.) Hassk & Florecilla & 2 & 2 & 2 & 1 & 2 & 2 \\
\hline & Tridax procumbens Humbert & Cadillo & 1 & 1 & 0 & 0 & 1 & 1 \\
\hline Commelinaceae & Murdania nudiflora (L.) Brenan & Cangrejillo & 1 & 1 & 1 & 0 & 2 & 1 \\
\hline \multirow[t]{3}{*}{ Euphorbiaceae } & Caperonia palustris (L.) St. Hilarie & ie Caperonia & 1 & 0 & 0 & 0 & 0 & 0 \\
\hline & Chamaesyce hirta (L.) Millspaugh & h Lechosa & 1 & 1 & 1 & 0 & 1 & 0 \\
\hline & Phyllanthus niruri $\mathrm{L}$. & Tamarindillo & 1 & 2 & 0 & 1 & 1 & 3 \\
\hline Lythraceae & Ammania coccinea Rottboell & Botoncillo & 1 & 1 & 2 & 1 & 4 & 1 \\
\hline Malvaceae & Sida acuta Burman F. & Escobilla & 1 & 2 & 0 & 0 & 1 & 3 \\
\hline Onagraceae & Ludwigia spp. & Clavito & 2 & 2 & 3 & 2 & 6 & 4 \\
\hline Pontederiaceae & Heteranthera limosa (Sw.) Willd. & Buche de gallina & 6 & 3 & 4 & 1 & 14 & 7 \\
\hline
\end{tabular}

* Entre 0 y $40 \mathrm{~cm}$ de profundidad. 
Fimbristylis); y hojas anchas, con 10 tipos diferentes de malezas en siete familias botánicas.

El arroz rojo junto con el grupo de las ciperáceas fueron las malezas con mayor importancia relativa, constituyendo el 66 y $76 \%$ del tamaño del banco de semillas del suelo en SDR y LMC, respectivamente. Estas malezas en el mismo orden respectivo sumaron el 77 y $89 \%$ de las plántulas detectadas en las pruebas de germinación en potes; e integraron casi la mitad de las malezas emergidas en el agroecosistema arrocero. Aunque en el grupo de hojas anchas hubo mayor diversidad de malezas, éstas integraron menos de una tercera parte del total, con mayor aporte de Eclipta alba, Ludwigia y Heteranthera limosa. En el grupo de gramíneas, Echinochloa colona fue la maleza con mayor importancia relativa, después del arroz rojo.

\section{Relación entre sistemas de labranza y dinámica de población de malezas}

En los cuatro ciclos de cultivo evaluados el banco de semillas del suelo en LMC fue mayor al de SDR $(\mathrm{p} \leq 0,05)$. Mientras que en SDR el tamaño total de este banco se estimó entre 3.500 y 3.700 semillas $/ \mathrm{m}^{2}$, en LMC fluctuó entre 3.800 y 4.700 semillas $/ \mathrm{m}^{2}$. La Figura 1 presenta los cambios observados en la composición del banco de semillas total en el transcurso de cuatro ciclos de evaluación. En SDR las proporciones de gramíneas y malezas de hojas anchas presentaron tendencias decrecientes, al contrario de las ciperáceas cuyo incremento mantuvo relativamente estable el tamaño total del banco. En LMC se observó una tendencia decreciente en las malezas de hojas anchas, mientras que las gramíneas y ciperáceas mostraron incrementos continuos.

\section{Tamaño del banco de semillas del suelo}

Cada sistema de labranza afectó en distinta forma la dinámica de diferentes poblaciones de malezas, favo-

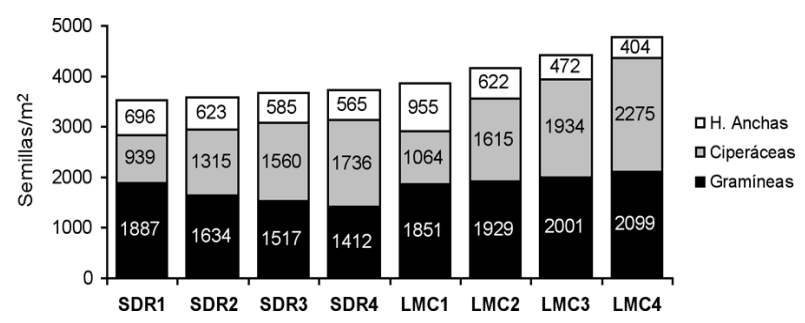

Figura 1. Cambios en el banco de semillas del suelo (0 a $40 \mathrm{~cm}$ de profundidad) según grupos de malezas y sistema de siembra. Hacienda El Pelón de la Bajura, Guanacaste, Costa Rica. 2004. reciendo el desarrollo de grupos particulares. El efecto de las labranzas en la composición del banco de semillas y la dinámica poblacional de las malezas es complejo e involucra varios factores (Tanaka y Anderson 2002). El Cuadro 2 presenta cambios observados en el tamaño del banco de semillas, en la germinación de semillas en potes y de malezas emergidas en el campo. Se incluyeron las dos especies de malezas más abundantes en los grupos gramíneas, ciperáceas y hojas anchas, durante cuatro ciclos consecutivos de evaluación. Aunque a gran escala la distribución espacial del banco de semillas del suelo se presenta en forma uniforme, a pequeña escala ésta es muy irregular dependiendo de la localización de las plantas madres y de la influencia relativa de los distintos agentes de dispersión, especialmente de los que actúan favoreciendo el movimiento superficial de las semillas.

En SDR hubo una gradual reducción en el tamaño del banco de semillas de arroz rojo. Después de los cuatro ciclos el tamaño del banco se redujo en $38 \%$ con respecto al tamaño inicial, mientras que aumentó $72 \%$ para las ciperáceas y $33 \%$ para hojas anchas anuales identificadas. Estudios previos (Buhler et al. 1998; Derksen y Lafond 2002) han demostrado que cuando se adopta la SDR las comunidades de malezas pueden cambiar. En general bajo SDR las malezas perennes, las gramíneas anuales y especies con semillas dispersadas por el viento incrementan, mientras que las hojas anchas anuales decrecen. Por el contrario, luego de cuatro ciclos en LMC el banco de semillas del arroz rojo incrementó en un $40 \%$ su tamaño inicial. En este sistema hubo además incrementos altamente significativos (100\% y $150 \%$ respectivamente) en los bancos de malezas ciperáceas anuales como Cyperus, Fimbristylis, con tasas de cambio superiores a las observadas en SDR. El sistema LMC favoreció reducciones del 30 y $69 \%$ en el tamaño de los bancos de las malezas Echinochloa colona y Heteranthera limosa.

En SDR la reducción acelerada de las semillas de arroz rojo que quedan sobre la superficie del suelo y no son incorporadas puede deberse a que quedan expuestas a condiciones adecuadas para su germinación, pérdida de viabilidad y depredación por animales, reduciéndose el potencial de semillas aptas a renovar la población de arroz rojo en el ciclo siguiente. En tales circunstancias el banco de semillas se empobrece continuamente con las semillas que desaparecen o mueren, con las que germinan y son controladas, o porque no todas las semillas que germinan producen plántulas viables en condiciones naturales. En algunos casos después de la cosecha la semilla de arroz entra en dormancia (Takahashi 1995; Vaughan et al. 1999) y son deterioradas por su contacto con patógenos del suelo. 
Cuadro 2. Banco de semillas del suelo, germinación en invernadero y malezas emergidas en el campo, según ciclo de cultivo y sistema de siembra para las seis principales malezas identificadas. Hacienda El Pelón de la Bajura, Guanacaste, Costa Rica. 2004.

\begin{tabular}{|c|c|c|c|c|c|c|c|c|c|c|}
\hline \multirow[t]{3}{*}{ Especie } & \multirow[t]{3}{*}{ Ciclo } & \multicolumn{3}{|c|}{ Banco de semillas } & \multicolumn{3}{|c|}{ Germinación en potes } & \multicolumn{3}{|c|}{ Emergencia en campo } \\
\hline & & SDR & LMC & Signif. & SDR & LMC & Singif & SDR & LMC & Signif. \\
\hline & & \multicolumn{3}{|c|}{ (Semillas/m² de suelo) } & \multicolumn{6}{|c|}{ (Plántulas/m², germinadas o emergidas) } \\
\hline \multicolumn{11}{|l|}{ Gramíneas } \\
\hline Oryza sativa & 1 & 1.325 & 1.275 & n.s. & 192 & 179 & n.s. & 23 & 24 & n.s. \\
\hline \multirow[t]{3}{*}{ Arroz rojo } & 2 & 1.045 & 1.535 & $* *$ & 151 & 216 & $* *$ & 19 & 28 & $*$ \\
\hline & 3 & 920 & 1.685 & $* *$ & 105 & 285 & $* *$ & 13 & 33 & $* *$ \\
\hline & 4 & 815 & 1.785 & $* *$ & 65 & 399 & $* *$ & 9 & 38 & $* *$ \\
\hline Echinochloa colona & 1 & 327 & 309 & $*$ & 26 & 21 & n.s. & 13 & 12 & n.s. \\
\hline \multirow[t]{3}{*}{ Arrocillo } & 2 & 320 & 247 & $*$ & 25 & 17 & $*$ & 9 & 10 & n.s. \\
\hline & 3 & 314 & 180 & $* *$ & 24 & 10 & $* *$ & 6 & 9 & n.s. \\
\hline & 4 & 310 & 215 & $* *$ & 23 & 7 & $* *$ & 4 & 8 & $*$ \\
\hline \multicolumn{11}{|l|}{ Ciperáceas } \\
\hline Cyperus spp. & 1 & 681 & 762 & n.s. & 149 & 167 & $*$ & 11 & 12 & n.s. \\
\hline \multirow[t]{3}{*}{ Coyolillos } & 2 & 920 & 1090 & $*$ & 201 & 239 & $*$ & 15 & 16 & n.s. \\
\hline & 3 & 1060 & 1276 & $* *$ & 313 & 400 & $* *$ & 17 & 24 & $*$ \\
\hline & 4 & 1176 & 1520 & $* *$ & 541 & 798 & $* *$ & 19 & 36 & $* *$ \\
\hline Fimbristylis milliaceae & 1 & 258 & 302 & n.s. & 44 & 51 & n.s. & 4 & 6 & n.s. \\
\hline \multirow[t]{3}{*}{ Pelo de chino } & 2 & 395 & 525 & $* *$ & 67 & 89 & $*$ & 7 & 9 & n.s. \\
\hline & 3 & 500 & 658 & $* *$ & 131 & 193 & $* *$ & 11 & 13 & n.s. \\
\hline & 4 & 560 & 755 & $* *$ & 283 & 483 & $* *$ & 20 & 15 & $*$ \\
\hline \multicolumn{11}{|l|}{ Hojas anchas } \\
\hline Ludwigia spp. & 1 & 54 & 91 & n.s. & 9 & 14 & n.s. & 2 & 7 & n.s. \\
\hline \multirow{3}{*}{ Clavito } & 2 & 75 & 100 & $* *$ & 13 & 15 & n.s. & 4 & 5 & $*$ \\
\hline & 3 & 88 & 103 & $* *$ & 20 & 17 & n.s. & 6 & 3 & $* *$ \\
\hline & 4 & 94 & 102 & $* *$ & 35 & 20 & $* *$ & 7 & 3 & $* *$ \\
\hline Heteranthera limosa & 1 & 181 & 223 & $* *$ & 23 & 27 & n.s. & 9 & 13 & n.s. \\
\hline \multirow[t]{3}{*}{ Buche de gallina } & 2 & 201 & 130 & $* *$ & 26 & 16 & $*$ & 11 & 8 & n.s. \\
\hline & 3 & 211 & 95 & $* *$ & 30 & 7 & $* *$ & 13 & 5 & $* *$ \\
\hline & 4 & 220 & 69 & $* *$ & 36 & 2 & $* *$ & 13 & 2 & $* *$ \\
\hline
\end{tabular}

Las diferencias entre datos fueron significativos a un nivel de $* 0,05$, o $0,01 * *$. n.s. es no significante a un nivel de 0,05 .

También las semillas caídas en los rastrojos pueden perderse por la depredación de aves, mamíferos e insectos, principalmente hormigas.

En SDR el banco de arroz rojo también puede disminuir porque como parte del sistema se estimula la germinación de semillas de ésta y otras malezas inundando los campos y aplicando herbicidas sistémicos en presiembra, especialmente glifosato, para destruir las malezas antes de que sus plántulas se reproduzcan y alimenten al banco con nueva semilla. Para lograr efectos significativos en el banco es indispensable que el control químico de las malezas sea altamente efectivo, pues una sola planta de arroz rojo con una producción de 900 granos y escapes de un 5\% es suficiente para reestablecer la cantidad de semillas del banco a su nivel original, en su vencidad.
Según Nolding (1995), las semillas de arroz rojo depositadas en la superficie del suelo pierden su viabilidad rápidamente. Después de la cosecha del arroz comercial, en las áreas infestadas con dicha maleza deben evitarse prácticas de manejo que entierren las semillas pues si éstas son depositadas a mayores profundidades se incrementa su longevidad. En terrenos sin rastrear ni plantar, el número de semillas viables de arroz rojo se reduce un $90 \%$ el primer año y $98 \%$ en dos años. Otra forma de disminuir el banco de arroz rojo es mantener el terreno inundado con una lámina de agua de 2 a $3 \mathrm{~cm}$ de altura. Un estudio realizado por Vidotto y Ferrero (2000) mostró que mientras en suelos saturados la germinación fue del $54 \%$, donde se mantuvo inundación continua sólo hubo una emergencia del $10 \%$ del arroz rojo procedente de semillas entre $0-10 \mathrm{~cm}$ de profundidad. 
La cobertura de rastrojos en SDR puede obstaculizar la germinación y emergencia de distintas malezas (Besnier 1989). En general altos niveles de residuos reducen la germinación y retardan la emergencia de plántulas germinadas, posiblemente por un decrecimiento de la amplitud térmica y disminución de la penetración de la luz (Dyer 1995). La composición espectral de la luz que penetra en el suelo cambia mucho en comparación con la luz solar. En los suelos arcillosos, típicos del sitio en estudio, la relación entre la luz roja y la luz rojo oscura es cercana a la unidad. En terrenos bajo SDR la cobertura de rastrojos filtra la luz y la relación entre luz roja/rojo oscura cae hasta valores menores de 0,1 en la superficie del suelo. Esto significa que la luz que penetra en el suelo es fundamentalmente rojo oscura, la cual produce la desactivación de la dormancia e induce a dormancia secundaria a las semillas que están en reposo.

Debido a que en SDR los rastrojos se mantienen sobre el suelo sin ser incorporados, la mineralización de la materia orgánica ocurre con mayor lentitud y limita la concentración de nitratos en el perfil del suelo. El nitrato es requerido por diversas especies de malezas para desactivar la dormancia y su concentración es considerada una señal ambiental que determina la emergencia estacional de algunas malezas. En SDR los residuos vegetales progresivamente se van acumulando sobre la superficie del suelo, y en su descomposición pueden inmovilizar grandes cantidades de nitrógeno, lo que podría resultar en un bajo contenido de nitrato en el suelo y evitar de ese modo que cese la dormancia en algunas malezas como es el caso del arroz rojo.

En LMC el arado y la rastra incorporan las semillas de arroz rojo y de otras malezas al interior del suelo, activando su dormancia y sometiéndolas a una latencia inducida (Acosta y Agüero 2001). A diferencia de las variedades cultivadas, las semillas de arroz rojo muestran distintos grados de dormancia. Algunas fuentes reportan entre uno a cinco años; según Diarra et al. (1985), la longevidad del arroz rojo puede ser hasta 12 años. En un estudio realizado en Estados Unidos, semilla de diferentes poblaciones de arroz rojo permaneció viable en un $90 \%$ después de dos años de enterrada y hasta un 20\% después de siete años (Goss y Brown 1939).

Subsecuentes operaciones de labranza en LMC vuelven a colocar las semillas enterradas en la superficie del terreno o cerca de ésta, donde las condiciones son propicias para la germinación y emergencia de la mayoría de las malezas. En varias especies la desactivación de la dormancia se completa sólo después que las semillas han sido expuestas a fluctuaciones de temperatura. La luz es otro factor que influye en la desactivación de la dormancia, lo cual generalmente ocurre cuando se llevan a cabo prácticas de labranza que expo- nen los fitocromos de semillas sensibles a períodos cortos e intermitentes de luz. La exposición de semillas a mayor intensidad y diferente calidad de luz, tiende a reducir la latencia de las semillas y promover su germinación (Swanton y Shestha 2002). La labranza del terreno también puede estimular la germinación de semillas al incrementarse el contacto entre éstas y el suelo, favoreciendo además la aeración (Buhler et al. 1998). El incremento observado en el tamaño del banco de semillas de arroz rojo en LMC puede ser explicado como un efecto resultante de la acción particular o combinada de los factores antes mencionados.

El incremento observado en las poblaciones de ciperáceas anuales, especialmente en Cyperus iria y Fimbristylis miliaceae $\mathrm{C}$. pudo deberse a que estas malezas alcanzan su período reproductivo mucho antes que el cultivo y se han adaptado para proliferar en ambientes acuáticos. Las mayores densidades observadas en LMC son consecuencia de la distribución horizontal uniforme de estas semillas por efecto de la labranza. Mientras que en SDR estas malezas se presentaron en forma de parches aislados, en LMC se distribuyeron en forma regular a través del campo.

Por otra parte, el sistema LMC ejerce efecto supresor sobre bancos de malezas anuales con semillas pequeñas como las de hoja ancha y gramíneas, distintas al arroz rojo identificadas en esta investigación. En este sistema las semillas pequeñas que quedan enterradas en forma profunda tienen menor probabilidad de germinar y emerger (Buhler 1998). La necesidad de luz que tienen muchas semillas para romper con su latencia, constituye un mecanismo para impedir la germinación a mayor profundidad. En las semillas enterradas, las pérdidas por ataques se reducen mucho, pero algunas semillas pueden sufrir ataques por diversas clases de organismos, incluso nemátodos. Las semillas enterradas en el suelo perecen por pérdida de viabilidad o por ataques de hongos y en menor grado por bacterias. Esta puede ser una de las razones por las cuales las poblaciones de malezas como Echinochloa y Heteranthera mostraron tendencias decrecientes.

\section{Germinación de malezas en potes}

En arroz rojo la proporción de plántulas que germinaron y emergieron con respecto al tamaño del banco de semillas fue relativamente baja. Durante los cuatro ciclos evaluados varió entre 8 y $14 \%$ en SDR y entre 14 y $22 \%$ en LMC, según los datos del Cuadro 2. Aunque la evaluación física practicada a semillas extraídas del banco reveló que prácticamente la totalidad de las mismas eran viables, estos bajos porcentajes pueden ser evidencia de la característica dormancia en semillas de 
esta maleza. Según Forcella (1992) varios estudios muestran que existe alta correlación entre el banco de semillas de arroz rojo, la emergencia en el suelo y el tipo de práctica de labranza adoptada. Típicamente la emergencia de malezas del banco de semillas puede oscilar desde 0,1 hasta $30 \%$.

Ferrero (2003) encontró que la emergencia de arroz rojo después de la preparación del suelo con arado de vertedera y mínima labranza fue de 2,5 y $7,2 \%$ respectivamente, del número de semillas presentes de 0 a $10 \mathrm{~cm}$ de profundidad. La diferencia entre estos valores se debió al movimiento de semillas de arroz ocasionadas por el arado. Con la inversión de la capa superficial del suelo se entierran semillas de producción reciente, estimulando su dormancia. Al mismo tiempo la rastra extrae semillas enterradas de ciclos anteriores, y las deja cerca de la superficie del suelo, aunque muchas de estas semillas han perdido su habilidad para germinar y muchas de las que germinan no logran emerger.

La germinación y emergencia de semillas de ciperáceas anuales colocadas en potes presentaron valores porcentuales más altos que los de arroz rojo. Aunque no se observaron diferencias entre sistemas de siembra, la germinación de especies del género Cyperus fue entre el 22 y $46 \%$ del total de semillas del banco, mientras que en Fimbristylis la proporción varió entre 16 y $64 \%$. Similar comportamiento presentaron las malezas de hojas anchas anuales, especialmente Eclipta alba, Phyllanthus niruri, Ludwigia spp. y Heteranthera limosa. Por el contrario, en las gramíneas anuales restantes la germinación fue de apenas entre 3 y $8 \%$ (Cuadro 2). Estas variaciones corresponden a distintas estrategias competitivas de las malezas para colonizar y competir en el agroecosistema.

\section{Malezas emergidas en el campo}

Se sabe muy poco sobre la proporción de semillas que en situaciones naturales germinan cada año. En el caso de semillas de malezas en terrenos cultivados, hay estudios que fijan esta proporción entre 1 y $4 \%$ de la población total hasta los $10 \mathrm{~cm}$ de profundidad (Monquero y Cristoffoletti 2003). Venoit y Lemieux (1987) estiman que solamente 0,3 a $9 \%$ de las semillas viables producidas desarrollan plántulas en un año dado y esta proporción se mantiene relativamente estable año tras año. En general cuando el número de semillas por planta adulta está regulado por la densidad de población, el banco de semillas del suelo sufre una oscilación amortiguada y termina estabilizándose, de no mediar accidentes catastróficos o la intervención humana. En el presente estudio las densidades de plántulas emergidas en el campo de las distintas malezas identificadas mostraron asociaciones directamente proporcionales al tamaño de los respectivos bancos. Concordando con los rangos antes mencionados, esta proporción varió entre 1 y $7 \%$ del tamaño del banco estimado en las muestras de suelo (Cuadro 2). Conforme se acentuaron cambios a lo largo del tiempo, especialmente en los últimos ciclos se observaron diferencias significativas en las poblaciones de malezas emergidas entre sistemas de siembra.

\section{Distribución vertical del banco de semillas de malezas}

La distribución vertical del banco de semillas influye en la capacidad de germinación y emergencia de las malezas. Mientras que en LMC la distribución de semillas de malezas es uniforme a través de diferentes rangos de profundidad del suelo, en terrenos no labrados la mayoría de las semillas se localizan en los primeros $5 \mathrm{~cm}$ de profundidad por debajo de la superficie, concentrándose en los dos primeros. Descendiendo a partir de los $5 \mathrm{~cm}$ de profundidad la población de semillas disminuye fuertemente, y la profundidad máxima que se alcanza depende de las características del suelo y de la vegetación de cobertura. Según Buhler (1998) en SDR más del 60\% se encuentra entre la superficie y $1 \mathrm{~cm}$ de profundidad. Cuando no hay labranza del suelo las semillas permanecen cerca de la superficie donde las más pequeñas tienen mayor facilidad para germinar, establecerse y proliferar, al contrario de lo que sucede con las de mayor tamaño.

Debido a que no hubo diferencias a lo largo del tiempo en la distribución vertical del banco de semillas de arroz rojo, en la Figura 2 se presentan los promedios correspondientes a datos acumulados en los cuatro ciclos evaluados. La respectiva distribución en las tres profundidades examinadas $(055 \mathrm{~cm}, 5-20 \mathrm{~cm}$ y 20 $40 \mathrm{~cm}$ ), fue de $62 \%, 25 \%$ y $13 \%$ para SDR y $36 \%, 31 \%$ y $33 \%$ en LMC, ajustándose al patrón señalado en estudios previos. Con respecto a la germinación en potes, en ambos sistemas de siembra se observó una significativa reducción en las muestras tomadas a mayor profundidad. Mientras que en LMC la distribución relativa con respecto al total de plántulas registradas fue de $62 \%, 23 \%$ y $6 \%$; lo correspondiente en SDR fue más acentuado, con $82 \%, 12 \%$ y $6 \%$, respectivamente. La longevidad de las semillas de arroz rojo está relacionada con la profundidad a la que se encuentran en el suelo y las prácticas de cultivo (Isihy y Noldin 1997).

Las propiedades físicas de los suelos afectan la distribución vertical de semillas de malezas. Existe evidencia de que el tipo de labranza tiene efectos diferenciales en la profundidad de la semilla a través de diferentes tipos de suelos. Por ejemplo, en un suelo arcilloso laborado 


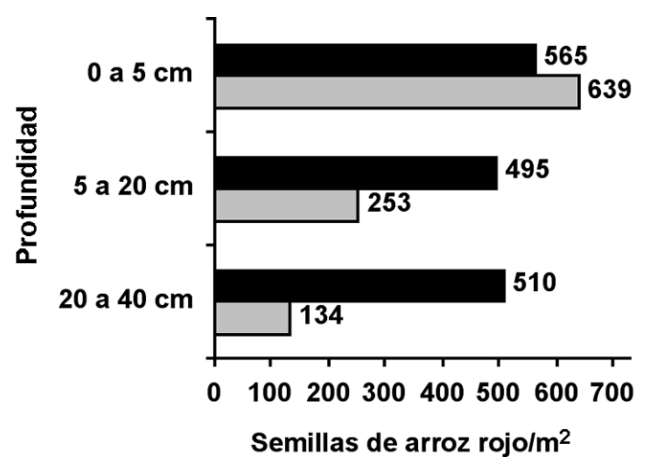

A- Banco total de semillas

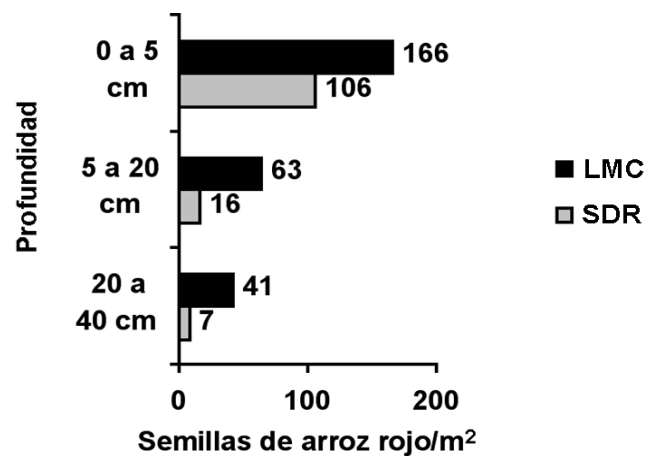

B- Germinación en potes

Figura 2. Distribución vertical del banco de semillas de arroz rojo y de su germinación en potes. Hacienda El Pelón de la Bajura, Guanacaste, Costa Rica. 2004.

con arado de vertedera $46 \%$ de las semillas estaban presentes en los $5 \mathrm{~cm}$ superficiales (Swanton et al. 2001). A esa misma profundidad, Clemens et al. (1996) reportaron contenidos de semillas del 37 y $74 \%$ bajo LMC y SDR, respectivamente. Pareja et al. (1985) observaron que la labranza convencional incorporaba semillas de malezas uniformemente en varias clases de agregados de suelos. En terrenos arcillosos la distribución de semillas cuando se usó el arado de vertedera fue más o menos uniforme en los primeros $15 \mathrm{~cm}$ de profundidad (Swanton et al. 2001).

En terrenos arados, la distribución vertical depende del tipo de instrumento que se utilice en la preparación del mismo para la siembra. La labranza entierra las semillas recién caídas y saca a la superficie semillas enterradas en temporadas anteriores, colocándolas en condiciones favorables para su germinación. En suelos no perturbados la distribución es más uniforme, aunque depende del momento en que se tome la muestra, pues después de la época principal de diseminación y antes de una operación de labranza hay una gran población superficial. A profundidades mayores que las de la capa arable, las poblaciones de semillas son muy escasas, y éstas sólo pueden ser llevadas a la superficie cuando se hacen actividades de subsolado a gran profundidad.

En suelos sin labrar las semillas alcanzan profundidades superiores a los $5 \mathrm{~cm}$ de diversas maneras. Semillas pequeñas, de longitud similar o menor a las del arroz, pueden ser arrastradas por el agua de lluvia o riego a través de las fisuras abiertas en suelos arcillosos durante los ciclos de humectación y desecación, o bien por los huecos dejados por las raíces descompuestas. Helpert (1986), constató que el $12 \%$ y $1 \%$ de las semi- llas de arroz rojo emergen desde 12 y $16 \mathrm{~cm}$ de profundidad, respectivamente. Muchas malezas poseen semillas con estructuras que facilitan su movimiento dentro del suelo. Por ejemplo, las semillas de arroz rojo poseen aristas y las de Echinochloa tienen picos estilares. De este modo se explica el $13 \%$ de granos de arroz rojo encontrados entre 20 y $40 \mathrm{~cm}$ de profundidad, aunque por lo general en los terrenos no labrados las profundidades máximas alcanzadas por las semillas no superan los $35 \mathrm{~cm}$. También es posible que las semillas enterradas a más de $20 \mathrm{~cm}$ de profundidad provengan de cultivos anteriores con labores mecanizadas.

\section{CONCLUSIÓN}

Aunque nuevos métodos de control químico selectivo estarán disponibles, seguirá siendo necesario combinar e integrar tácticas para manejar las poblaciones de malezas en el cultivo, en especial cuando éstas se le asemejan fisiológica y físicamente, como es el caso del arroz rojo y el arroz comercial. Los resultados de esta investigación demostraron que tanto para el arroz rojo como en las malezas predominantes del agroecosistema arrocero, el sistema de preparación del suelo para la siembra del cultivo afectó las poblaciones del banco de semillas del suelo, su distribución vertical, la germinación y la emergencia de poblaciones en el campo. En comparación con la LMC, la SDR facilitó el control de arroz rojo y otras malezas asociadas al cultivo de arroz. Adicionalmente la SDR permitió ahorros significativos en los costos de preparación del suelo y puede contribuir substancialmente a la calidad de los recursos naturales mediante la reducción de la erosión. 
Para integrar apropiadamente la SDR a programas de manejo holístico de agroecosistemas, se requiere conocimiento de la ecología de la población de las malezas y poder predecir las respuestas a los cambios en las prácticas de manejo. En un contexto amplio para comprender la dinámica de la población de malezas como un todo, son requeridos estudios empíricos del impacto de diferentes tipos de labranza, manejo del agua y aplicaciones de herbicidas. También es particularmente importante medir la longevidad de las semillas en el suelo y el impacto de las prácticas de labranza.

\section{LITERATURA CITADA}

ACOSTA, L.; AGÜERO, R. 2001. El banco de propágulos de malezas en el agroecosistema: Conocimiento actual y propuesta metodológica para su estudio. Agronomía Mesoamericana 12 (2): 141-151.

AGÜERO; R. 1996. Malezas del arroz y su manejo. Centro de Investigaciones en Protección de Cultivos, Universidad de Costa Rica. 246 p.

BAKER, J.B.; SONNIER, E.A. 1983. Red rice and its control. In: Weed control in rice. IRRI. p. 327-333.

BENECH-ARNOLD, R.L ; SÁNCHEZ, R.A.; FORCELLA, F. ; KRUK, B.C.; GHERSA, C.M. 2000. Environmental control of dormancy in weed seed banks in soil. Field Crops Research 67:105-122.

BESNIER; R. 1989. Semillas: biología y tecnología. Ed. Mundi-prensa, Madrid. 637 p.

BUHLER; D.D. 1998. Adjust weed control strategy for conservation tillage. Weed Control Manual. p. 68-69.

BUHLER, D.D.; HARTZLER, R.G.; FORCELLA, F. 1998. Weed seed bank dynamics implications to weed management. Crop Science. p. 145-168.

CLEMENTS, D.R.; BENOIT, D.L; SWANTON, C.J. 1996. Tillage effects on weed seed return and seedbank composition. Weed Science 44:314-322.

CONOVER, D.G.; GEIGER, D.R. 1984. Germination of australian channel millet Echinochloa turneriana (Domin) J.M. Black seeds. Effects of anaerobic conditions, continuous flooding, and low water potential. Australian Journal of Plant Physiology 11:409-417.

DERKSEN, D.A.; LAFOND, G.P. 2002. Weed community changes and conservation-tillage systems (en línea). Consultado 17 mayo 2004. Disponible en http://paridss.usask.ca/facbook/soilcrop/derksen1.html.
DIARRA, A.; SMITH, R.J.; TALBERT, R.E. 1985. Red rice (Oryza sativa L.) control in drill-seeded rice $(O$. sativa). Weed Science 33:703-707.

DYER, W. E. 1995. Exploiting weed seed dormancy and germination requirements through agronomic practices. Weed Science 43(3): 498-503.

FERRERO, A. 2003. Weedy rice, biological features and control. In: Weed Management for Developing Countries. FAO Plant Production and Protection paper 120. 18 p.

FISHER, A.J. 1999. Problems and opportunities for managing red rice in Latin America. In: Taller Global de Control de Arroz Rojo. Cuba, p. 77-85.

FISHER, A.J.; RAMIREZ, A. 1993. Red rice (Oryza sativa): competition studies for management decisions. International Journal of Pest Management 39: 133-138.

FORCELLA, F. 1992. Prediction of weed seedling densities from buried seed reserves. Weed Res 32: 29-38.

GONZALEZ, J. 1985. El arroz rojo y su control. In: Arroz: investigación y producción. CIAT. Cali, Colombia. p. 459-475.

GOSS, W.L.; BROWN, E. 1939. Buried red rice. Journal. of American Society of Agronomy 31: 633-637.

HELPERT, C. W. 1986. Dormancy, germination, and emergence of red rice (Oryza sativa L.). Ph.D. Thesis. Texas, Texas A\&M University, Texas. 92 p.

INSAUSTI, P.; SORIANO, A.; SÁNCHEZ, R.A. 1995. Effects of flood-influenced factors on seed germination of Ambrosia tenuifolia. Oeologia 103:127-132.

ISIHY, T.; NOLDIN, J.A. 1997. Controle de misturas varietais atraves de agua de irrigacao na cultura do arroz irrigado sistema pre germinado. Annais da Cultura do Arroz Irrigado (Itajai) 22: 478-480.

KEGODE, G.O.; FORCELLA, F.; CLAY, S. 1999. Influence of crop rotation, tillage, and management inputs on weed seed production. Weed Science 47: 175-183.

KHUSH, G.S.; BENNET, J.; DATTA, S.K.; BRAR, D.S. LI Z. 1999. Advances in rice genetics and biotechnology. In: International Rice Commission Newsletter. FAO. Roma. (en línea). Consultado 19 jun. 2004. Disponible en: http://www.fao.org/DOCREP/ 003/ X2243T/x2243t04.htm\#P4_166.

KWON, S.L.; SMITH, R.J.; TALBERT, R.E. 1991. Interference of red rice ( $O$. sativa) densities in rice $(O$. sativa). Weed Sci. 39: 169-174. 
MONQUERO, P.A.; CHRISTOFFOLETI, P.J. 2003. Seed bank dynamics in areas with frequent glyphosate application. Planta daninha 21(1):63-69.

MOODY, K. 1991. Weed management in rice. In: Pimentel D. (ed). Handbook of pest management in agriculture. $2^{\text {nd }}$ ed. Boca Raton, Florida: CRC Press, Inc p. 301-328.

MORTIMER, M.; PANDEY, S.; PIGGIN, C. 2000. Weedy rice: approaches to ecological appraisal and implications for research priorities. In: Wild and weedy rice in rice ecosystems in Asia - A review. IRRI. p. 97-105.

NOLDIN, J.A. 1995. Characterization, seed longevity and herbicide sensitivity of red rice (Oryza sativa $\mathrm{L}$ ) ecotypes, and red rice control in soybeans (Glycine max). Texas,Texas A\&M, University. 218 p.

OLOFSDOTTER, M.; VALVERDE, B.E.; MADSEN, K.H. 1999. Herbicide resistant rice (Oryza sativa L.) - A threat or a solution. International Rice Research Institute, Philippines In: Taller Global de Control de Arroz Rojo. Cuba, p. 123 - 142.

PANTONE, D. BAKER, J. 1991. Reciprocal yield analysis of red rice (Oryza sativa) competition in cultivated rice. Weed Sci. 39: 42-47.

PAREJA, M. R.; STANIFORTH, D. W.; PAREJA, G. P. 1985. Distribution of weed seed among soil structural units. Weed Science 33:182-189.

ROSA, M.L.; CORBINEAU, F. 1986. Quelques aspects de la germination des caryopses de Leersia oryzoides (L.) Sw. Weed Research 26:99-104.

ZHANG, C.X. 2000. Wild and weedy rice in China. In: Wild and weedy rice in rice ecosystems in Asia - A review. Baki BB, Chin DV y Mortimer R. IRRI. Filipinas. p.35.

SMITH, R. J. 1988. Weed thresholds in southern US rice (Oryza sativa). Weed Technology. 2:232-241.
SWANTON, C.J.; SHRESTHA, A. 2002. Tillage, soil type and weed seed bank dynamics (en línea). Consultado 15 de mayo 2004. Disponible en: http://www.plant. uoguelph.ca/research/weedsci/seedbank.htm..

SWANTON, C.J.; SHRESTHA, A.; CHANDLER, K. 2001. Tillage, soil type, and weed seed bank dynamics. Ontario Corn Producers 28 p.

TAKAHASHI N. 1995. Physiology of dormancy. In: T. Matsuo, K. Kumazawa, R. Ishii, K. Ishihara, and H. Hirata (eds.). Science of the Rice Plant. Vol. 2. Physiology. Food and Agriculural Policy Research Center, Tokio. p. 45-57.

TANAKA, D. L.; ANDERSON, R. L. 2002. Weed community response to cultural practices rotation, tillage and nitrogen fertility. (en línea). Consultado 19 junio 2004. Disponible en: http://www.mandakzerotill.org/book18/ cultural.html.

VAUGHAN D.H. WATANABE H. LAMBERS H.R. ZAIN M.O. TOMOOKA N. 1999. Weedy rice complexes in direct seeding rice cultures. Proceedings of the International Symposium on World Food Security. p. 227-280.

VIDOTTO, F.; FERRERO, A. 2000. Germination behavior of red rice (Oryza sativa L.) seeds in field and laboratory conditions. Agronomie 20: 375-382.

WESTERMAN, P.R.; WES, J.S.; KROPFF, M.J. VAN DER WERF W. 2003. Annual losses of weed seeds due to predation in organic cereal fields. Journal of Applied Ecology 40: 824-836.

ZORRILLA, G.; ACEVEDO, A. 1996. Semillas: investigación y tecnología de semillas. In: Arroz. Resultados experimentales 1995-1996, 33 Uruguay, INNIA, Estación Experimental del Este. p. 1-6. 